PROCEEDINGS OF THE

AMERICAN MATHEMATICAL SOCIETY

Volume 128, Number 7, Pages 2095-2103

S 0002-9939(00)05526-X

Article electronically published on March 2, 2000

\title{
MINIMAL CURVES OF CONSTANT TORSION
}

\author{
THOMAS A. IVEY \\ (Communicated by Christopher Croke)
}

\begin{abstract}
The Griffiths formalism is applied to find constant torsion curves which are extremal for arclength with respect to variations preserving torsion, fixing the endpoints and the binormals at the endpoints. The critical curves are elastic rods of constant torsion, which are shown to not realize certain boundary conditions.
\end{abstract}

In the calculus of variations under nonholonomic constraints, one tries to find the least energy trajectory among solutions of a given differential equation. The subject has its roots in the investigations of such classical questions as Pappus' problem and the Delaunay problem [6]. It is a standard part of optimal control theory, where such problems are investigated using the Pontrjagin maximum principle [12]. In recent years, the subject has come to the attention of geometers again, with the investigations of sub-Riemannian geometry [13, but also with the arrival of a beautiful reformulation, due to Griffiths and his collaborators 3, 9, 10, of the criticality conditions in coordinate-free form.

The stated aim in Griffiths' 1983 book [9] is "to get out formulas" for critical curves. In this note, we carry this out for the problem of length minimization among curves in $\mathbb{R}^{3}$ of a fixed nonzero constant torsion. This is among the problems to which Hsu [10] applied the Griffiths formalism, but here we go further, giving complete formulas for critical curves, and are able to extract more information about what boundary values can and cannot be achieved. This is due to the observation that the critical curves for this problem coincide exactly with the subset of Kirchhoff elastic rod centerlines having constant torsion, and the detailed description of these centerlines by Langer and Singer [11].

The constant torsion constraint is arrived at by considering the general case. Suppose we adapt an oriented orthornormal frame $(T, U, V)$ along an oriented curve $\gamma$ in $\mathbb{R}^{3}$, parametrized by arclength $s$, such that $T$ is the unit tangent. The frame will satisfy generalized Frenet equations

$$
\frac{d}{d s}\left(\begin{array}{l}
T \\
U \\
V
\end{array}\right)=\left(\begin{array}{ccc}
0 & y & p \\
-y & 0 & r \\
-p & -r & 0
\end{array}\right)\left(\begin{array}{l}
T \\
U \\
V
\end{array}\right) .
$$

If these vectors are regarded as attached to a rigid object moving along $\gamma$, the functions $p, r, y$ may be visualized as pitch, roll, and yaw, respectively.

Received by the editors September 2, 1998.

2000 Mathematics Subject Classification. Primary 49K15, 53A04; Secondary 58A17, 58A30, $73 \mathrm{C} 02$

Key words and phrases. Curves of constant torsion, Griffiths formalism, elastic rods. 
Suppose two of $p, r, y$ are prescribed constants. Because $U$ and $V$ can be interchanged, we need only consider the $p \& r$ case and the $p \& y$ case. In the latter case, we can rotate $U$ and $V$ to arrange that $p=0$. In either case, the lift $(\gamma(s), T(s), U(s), V(s))$ into the oriented orthornomal frame bundle $\mathcal{F}^{6}$ is an integral curve of some given distribution of rank two, or equivalently, of a Pfaffian system $\mathcal{I}$ of rank four.

We may ask if any two points of $\mathcal{F}$ can be connected by an integral curve of the distribution. The answer is provided by Chow's theorem [7, which says that if a smooth constant rank Pfaffian system $\mathcal{I}$ on manifold $M$ contains no non-trivial Frobenius subsystem at any point, then any two points of $M$ may be connected by a continuous, piecewise smooth integral curve of $\mathcal{I} 1$ If this happens, we will say that $M$ is accessible under the constraint given by $\mathcal{I}$. On the other hand, if a Frobenius subsystem $\mathcal{J}$ is present, and the integral submanifolds of $\mathcal{J}$ form a non-trivial foliation of $M$, then connecting pairs of points on different leaves is impossible.

It is easy to check the condition in Chow's theorem in the two cases mentioned above.

Proposition 0.1. $\mathcal{F}$ is accessible under $(a) p=0, y$ a nonzero constant, and (b) $p$ constant, $r$ a nonzero constant. Under $p$ constant and $r=0$, the corresponding Pfaffian system contains a nontrivial Frobenius subsystem.

Frames with $p=0$ are of course the usual Frenet frames, with curvature $\kappa=y$ and torsion $\tau=r$. Frames with $r=0$ are called relatively parallel adapted frames by Bishop [1. If the curvatures $p(s)$ and $y(s)$ of such a frame satisfy $a p+b y=c$ for constants $a, b, c$, then the curve $\gamma$ lies on a sphere 2 of radius $\sqrt{a^{2}+b^{2}} / c$.

Length minimization in case (a) above is the classical Delaunay problem. (However, for the Delaunay problem one usually only prescribes the endpoints and at most the tangents, rather than the whole Frenet frame, at each end.) We will concentrate on the analogous question in case (b). There, the critical curves turn out to be the same no matter what value $p$ has (details here are left to the reader), so we will assume $p=0$. Then our problem is to minimize length among curves of fixed nonzero constant torsion. The reader should note that a straight line, equipped with a Frenet frame with $N$ and $B$ rotating around the line at a constant rate, counts as such a curve; we will refer to this as a "spinning straight line."

Our results are:

Theorem 1. Any smooth curve which is critical for arclength, with respect to variations among curves of a fixed nonzero constant torsion, fixing the Frenet frame at the endpoints, must be either a spinning straight line or an elastic rod centerline of constant torsion. The latter curves, which divide into two two-parameter families, are also critical with respect to variations that fix only the binormal B at both endpoints.

Theorem 2. None of these curves satisfy boundary conditions where the binormals at the endpoints are opposing vectors pointing along a common straight line, and the distance between the endpoints is less than $2 \pi / \tau$.

\footnotetext{
${ }^{1}$ The hypothesis in Chow's theorem is usually put as the "strong bracket-generating" condition for vector fields in the distribution; it is easy to verify that the dual formulation given here is equivalent.

${ }^{2}$ This result was apparently known to Enneper, and used in his investigations of surfaces of constant curvature with spherical lines of curvature; see [8], article 814. I thank I. Sterling for pointing this out.
} 
Several comments are in order regarding these two theorems and their proof (which takes up the remaining two sections of the paper).

First, these results should be compared with the classical results for the Delaunay problem, which may be found in Carathéodory's survey article [6]. Like the extremals for the Delaunay problem, which were completely determined by Weierstrass, our extremals will be expressed in terms of elliptic integrals. Here it should be noted that the Griffiths formalism is easily the most rapid way of obtaining the criticality conditions (cf. the treatment of the Delaunay problem on pp. 153-157 in 9]). It can also be used to investigate the second variation, although we have not done so here.

Under boundary conditions which only fix the endpoints of the curve, no absolute minimum exists for the Delaunay problem, while the minimum here is trivial (the spinning straight line). While the boundary conditions in Theorem 1 are of higher order than those usually imposed in the Delaunay problem, they are consistent with the constant torsion constraint, which is third order in the derivatives of the curve. The binormal boundary condition is also convenient because a constant torsion curve may be recovered from its binormal indicatrix, through the integral formula

$$
\gamma=\frac{1}{\tau} \int B \times \frac{d B}{d s} d s
$$

(see [ 8 , Note IV). Thus, one may turn the present problem into an isoperimetric problem, with fixed endpoints, for curves on the unit sphere. (In a similar way, H.A. Schwarz investigated the second variation for the Delaunay problem by passing to an isoperimetric problem for the tangent indicatrix.)

Because of Theorem 2 and the profusion of critical curves provided by Theorem 11 it would perhaps be appropriate to seek curves which are critical with respect to a larger class of variations among constant torsion curves - say, fixing the endpoints and the binormal at only one end. On the other hand, it is clear that the shortest constant torsion curves fulfilling the boundary conditions in Theorem 2 would, if they exist, include singular points. Numerical experiments (to be reported elsewhere), applying K. Brakke's program The Surface Evolver [2] to the isoperimetric version of the problem, seem to bear this out. It would be interesting to know the nature and the minimum number of these singular points.

\section{Application of the Griffiths formalism}

The Griffiths formalism provides a clean set of Euler-Lagrange conditions which are (most of the time) necessary and sufficient for criticality with respect to variations among constrained curves. Since these conditions are phrased in terms of differential forms, we will need a formulation of the Frenet equations and the constant torsion condition as a Pfaffian system on $\mathcal{F}$.

Labelling $T, N, B$ as vector-valued functions $e_{1}, e_{2}, e_{3}$ on $\mathcal{F}$, and $x$ as the basepoint function, there are differential 1 -forms $\omega^{1}, \omega^{2}, \omega^{3}, \phi_{2}^{3}, \phi_{3}^{1}, \phi_{1}^{2}$ on $\mathcal{F}$ such that

$$
d x=e_{i} \omega^{i}, \quad d e_{i}=e_{j} \phi_{i}^{j} .
$$

These 6 forms are everywhere linearly independent, and satisfy structure equations

$$
d \omega^{i}=-\phi_{j}^{i} \wedge \omega^{j}, \quad d \phi_{j}^{i}=-\phi_{k}^{i} \wedge \phi_{j}^{k} .
$$

\footnotetext{
${ }^{3}$ Here, the indices run from 1 to 3 , summation is implied by repeated indices, and $\phi_{j}^{i}=-\phi_{i}^{j}$.
} 
The Frenet frame of a general curve in $\mathbb{R}^{3}$ gives a lift into $\mathcal{F}$ which is an integral curve of $\omega^{2}, \omega^{3}$ and $\phi_{1}^{3}$. That of a curve of constant torsion $\tau$ gives an integral curve of the Pfaffian system

$$
\mathcal{I}=\left\{\omega^{2}, \omega^{3}, \phi_{1}^{3}, \phi_{2}^{3}-\tau \omega^{1}\right\} .
$$

Using differential forms allows us to formulate geometric conditions in a coordinate-free way. For example 4 here is how we can express the classical EulerLagrange equations in terms of forms. Suppose we fix a smooth 1-form $\omega$ defined on some manifold $M$, and we seek a curve with given endpoints $P$ and $Q$ that is critical for $\int \omega$. If $\gamma$ is such a curve, let $\tilde{\gamma}$ be a nearby curve with the same endpoints and let $\Omega$ be the disc between. Then, by Stokes' Theorem,

$$
\int_{\tilde{\gamma}} \omega-\int_{\gamma} \omega=\int_{\Omega} d \omega
$$

So, the infinitesimal variation of $\int_{\gamma} \omega$ along a transverse vector field $\mathrm{v}$ fixing the endpoints is

$$
\left.\delta_{\mathrm{v}} \int_{\gamma} \omega=\int_{\gamma} \mathrm{v}\right\lrcorner d \omega .
$$

Thus, for a critical curve $\left.\gamma, \int_{\gamma} \mathrm{v}\right\lrcorner d \omega$ should be zero for all possible transverse vector fields v. This gives a pointwise condition,

$$
\left.\gamma^{\prime}\right\lrcorner d \omega=0 .
$$

Suppose now that a smooth Pfaffian system $\mathcal{D}$ is defined on $M$, and we seek a curve between $P$ and $Q$ that is critical for $\int \omega$ among integral curves of $\mathcal{D}$. Suppose $\mathcal{D}$ has a local basis $\theta_{1}, \cdots, \theta_{n}$. The Griffiths condition resembles Lagrange multipliers: suppose there are functions $\lambda_{i}, 1 \leq i \leq n$, defined along a curve $\gamma$, such that $\gamma$ satisfies the usual Euler-Lagrange condition (3) with respect to the modified 1 -form $\tilde{\omega}=\omega-\sum \lambda_{i} \theta^{i}$. If $\mathrm{v}$ is tangent to a variation of $\gamma$ through integrals of $\mathcal{D}$, then

$$
\delta_{\mathrm{v}} \int_{\gamma} \omega=\delta_{\mathrm{v}} \int_{\gamma} \omega-\sum_{i} \lambda_{i} \delta_{\mathrm{v}} \int_{\gamma} \theta^{i}=\delta_{\mathrm{v}} \int_{\gamma} \tilde{\omega}=0 .
$$

For integrals of $\mathcal{I}$ in (2), arclength for the underlying curve in $\mathbb{R}^{3}$ is given by $\int \omega^{1}$. Pairing multiplier functions $\lambda_{1}, \lambda_{2}, \lambda_{3}, \lambda_{4}$ with 1 -forms $\phi_{1}^{3}+\tau \omega^{2}, \phi_{2}^{3}-\tau \omega^{1}$, $\omega^{3}, \omega^{2}$ respectively, we obtain the following Euler-Lagrange system:

$$
\begin{aligned}
& \frac{d \lambda_{1}}{d s}=\lambda_{3}\left(2 \tau \lambda_{2}-1\right), \quad \frac{d \lambda_{2}}{d s}=-2 \tau \lambda_{1} \lambda_{3}, \\
& \frac{d \lambda_{3}}{d s}=-\tau^{2} \lambda_{1}, \quad \lambda_{4}=0, \quad \kappa=2 \tau \lambda_{3} .
\end{aligned}
$$

Hsu [10] observed that $R^{2}=\left(\tau \lambda_{1}\right)^{2}+\left(\tau \lambda_{2}-\frac{1}{2}\right)^{2}$ is a first integral of these equations, and that helices result when $R=0$. What is new is the observation that the general solution of these equations, and the underlying curves, can be obtained explicitly. Introduce the change of variables $\tau \lambda_{1}=R \sin (2 t), \tau \lambda_{2}=R \cos (2 t)+\frac{1}{2}, R>0$, giving $d t=\tau \lambda_{3} d s$. Then $(d / d t)\left(\lambda_{3}{ }^{2}\right)=-2 R \sin (2 t)$ gives $\lambda_{3}{ }^{2}=2 R\left(C-\sin ^{2} t\right)$ for some positive constant $C$. The solutions now are of two types, depending on $C$.

\footnotetext{
${ }^{4}$ I am indebted to Robert Bryant for this description of the classical setup.
} 
Type 1. If $C \geq 1$, let $p=1 / \sqrt{C}$ and $a=\tau \sqrt{2 C R}$, whereupon

$$
\frac{d t}{d s}=a \sqrt{1-p^{2} \sin ^{2} t} .
$$

Then $t$ is given in terms of $s$ by inverting an elliptic integral 5

$$
t=\operatorname{am}(a s, p),
$$

and the curvature is given by an elliptic function of modulus $p$,

$$
\kappa=2 \tau \lambda_{3}=2 a \operatorname{dn}(a s, p) .
$$

Type 2. If $C<1$, let $p=\sqrt{C}, a$ as before, whereupon

$$
\left(\frac{d t}{d s}\right)^{2}=a^{2}\left(1-\frac{\sin ^{2} t}{p^{2}}\right)
$$

Now set $\sin t=p \sin \theta$, and $\frac{d t}{d s}=a \cos \theta$. Then

$$
\frac{d \theta}{d s}=\frac{a}{p} \sqrt{1-p^{2} \sin ^{2} \theta}
$$

giving $\theta=\operatorname{am}(a s / p, p)$ and $\kappa=2 a \operatorname{cn}(a s / p, p)$.

Because $\kappa$ is periodic in either case (except when $C=1$ ), the corresponding curves in $\mathbb{R}^{3}$ will be composed of a succession of congruent segments, fitting smoothly together. Their shape will depend on two dimensionless constants, $p$ and $\sigma=\tau / a$.

To know more about these curves, it is necessary to solve the Frenet equations, and integrate $T$. While in general it is difficult to find explicit solutions, in our case we observe that the curvature functions are precisely those of two families of elastic rod centerlines of constant torsion. From the work of Langer and Singer [11, we know there are natural cylindrical coordinates $(r, \theta, z)$ in which Type 1 curves satisfy

$$
\begin{aligned}
r^{2} & =\left(\operatorname{dn}^{2} u-\frac{\sigma^{2}\left(1-p^{2}\right)}{4 \mu^{2}}\right) /\left(4 a^{2} \mu^{2}\right), \quad u=a s, \\
\frac{d z}{d s} & =\frac{\mathrm{dn}^{2} u}{2 \mu}+\frac{p^{2}-\sigma^{2}-2}{4 \mu} \\
\frac{d \theta}{d u} & =L-\frac{N}{1-M \operatorname{sn}^{2} u},
\end{aligned}
$$

where the constants $\mu, L, M, N$ are given by $\mu=\sqrt{\left(p^{2}-\sigma^{2}\right)^{2}+4 \sigma^{2}} / 4, L=$ $\tau\left(p^{2}-\sigma^{2}-2\right) /(8 \mu), M=16 \mu^{2} p^{2} /\left(p^{2}+\sigma^{2}\right)^{2}, N=2 \mu \sigma /\left(p^{2}+\sigma^{2}\right)+L$. Similar formulas for Type 2 curves will be given below.

Figure 1 shows some examples of Type 1 curves. When $p \approx 0$, Type 1 curves are approximately helical. As $p$ increases, the ratio of maximum to minimum curvature increases, producing a succession of looser and tighter coils. Increasing $\sigma$ stretches out these coils. When $p=1$, corresponding to $C=1$, the curvature is given by a hyperbolic secant, and the curve is asymptotic to a straight line. (This particular elastic rod centerline is known as the Hasimoto filament.)

\footnotetext{
${ }^{5}$ Notations and definitions for elliptic functions and integrals are taken from [4].
} 
While it can be shown that Type 1 curves are never closed, Type 2 curves can be made to close up, and even to form knots (see Figure 2 on the next page). These closed curves of constant torsion are the starting point for the production of more exotic knots of constant torsion via Bäcklund transformations [5].

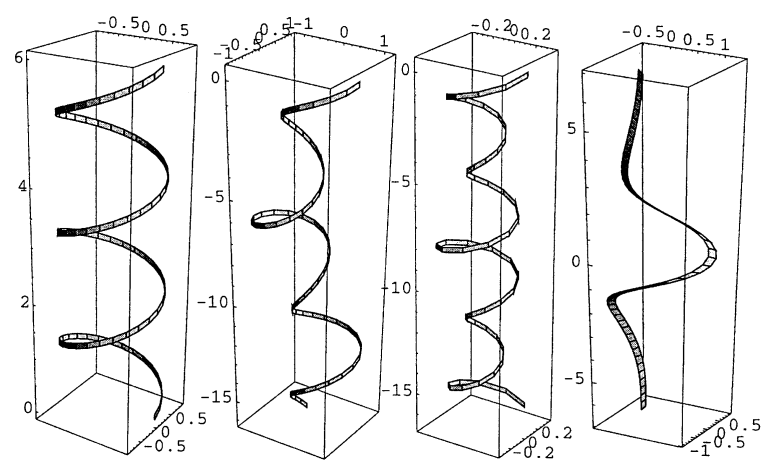

Figure 1. Some Type 1 minimal constant torsion curves. For the helix at left, $p=0$. In the middle two curves, $p=0.9$ and $\sigma=1.4$ and 3 respectively. At right is the Hasimoto filament.

\section{BOUNDARY CONDITIONS}

Although our explanation of the Griffiths condition indicates why the resulting curves are critical with respect to variations fixing the endpoints in $\mathcal{F}$, it turns out that more is true. According to Griffiths (9], p. 199), a solution to our EulerLagrange system (4) is critical with respect to all infinitesimal variations among integrals of $\mathcal{I}$ which satisfy $\mathrm{v}\lrcorner \mathcal{I}=0=\mathrm{v}\lrcorner \omega^{1}$ at the endpoints. This corresponds to variations that move along the fibre of $\mathcal{F}$ at the endpoints, fixing the binormal vector $B$. Thus, our extremal elastic rod centerlines of the last section are among the solutions of the problem:

What are the minimal curves of given constant torsion between two given points in $\mathbb{R}^{3}$, with given binormal vectors at the endpoints?

—provided by "minimal" we mean "critical for arclength," just as when we talk about minimal surfaces, by "minimal" we mean "critical for area."

It is not obvious that the Griffiths condition is necessary for all solutions of the above minimization problem. However, Hsu [10] has developed a notion of regularity for smooth integral curves of a Pfaffian system $\mathcal{I}$, such that regular curves that are critical, among other integrals, for a given Lagrangian, must arise from the Griffiths formalism. In our case, the only non-regular integrals of the system $\mathcal{I}$ in (2) are those tangent to the fibre of $\mathcal{F}$ (corresponding to sitting at a point in $\mathbb{R}^{3}$ and spinning the frame) and those along which $\phi_{1}^{2}=0$, corresponding to a spinning straight line. Thus, we conclude that, for boundary conditions for which a spinning straight line is not a solution, any smooth critical curve must be one of the elastic rod centerlines derived in the previous section.

Unfortunately, it turns out that not all boundary conditions are available from these curves. For Type 1 curves, the binormal is given by

$$
B=\left(\frac{\sigma p^{2} \operatorname{cn} u \operatorname{sn} u}{2 \mu^{2} r}, \frac{\sigma^{4}-p^{4}}{16 \mu^{3} r^{2}} \operatorname{dn} u,-\frac{\sigma \operatorname{dn} u}{2 \mu}\right) \cdot\left(\partial_{r}, \partial_{\theta}, \partial_{z}\right)
$$



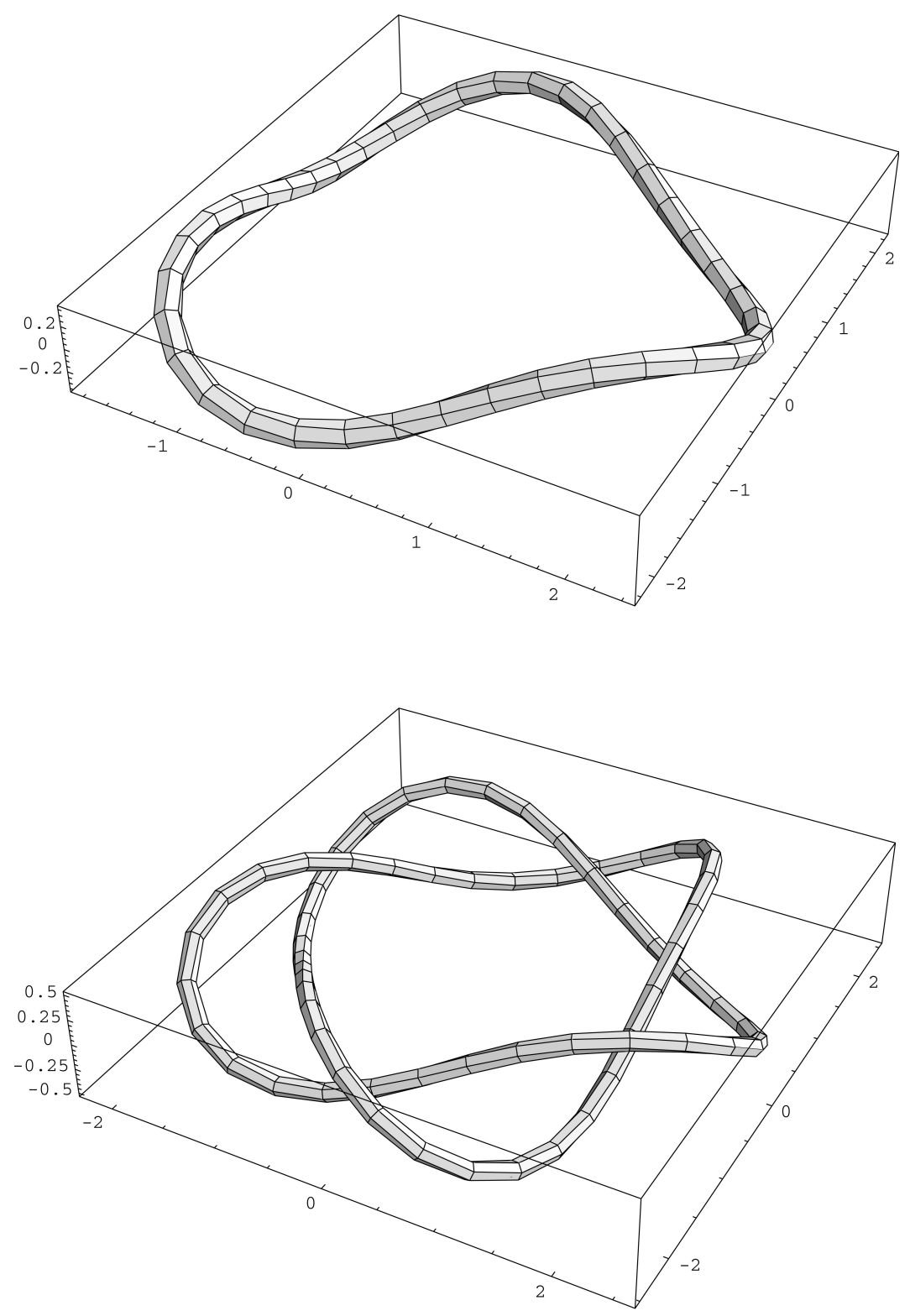

Figure 2. Some closed Type 2 minimal constant torsion curves

in terms of the cylindrical coordinate basis vectors. Consider boundary conditions where $B$ is required to point in opposite directions at the endpoints of the curve. Since $\operatorname{dn} u$ is strictly positive, this is impossible for Type 1 curves.

Suppose that our boundary conditions also require the binormals at the endpoints to be parallel to the straight line connecting the endpoints 6 For Type 2

${ }^{6}$ For spinning straight lines, this is impossible. 
curves,

$$
B=\left(\frac{\sigma \operatorname{sn} u \operatorname{dn} u}{2 p \mu^{2} r}, \frac{\left(\sigma^{4}-p^{-4}\right) \operatorname{cn} u}{16 \mu^{3} r^{2}},-\frac{\sigma \operatorname{cn} u}{2 \mu}\right) \cdot\left(\partial_{r}, \partial_{\theta}, \partial_{z}\right),
$$

where $u=a s / p$ and $\mu=\sqrt{\left(p^{-2}-\sigma^{2}\right)^{2}+4 \sigma^{2}} / 4$. Recall that $2 K$ is the antiperiod of cn $u$ ( $K=K(p)$ being the complete elliptic integral of the first kind). If $u_{1}$ and $u_{2}$ are parameter values corresponding to the endpoints, then examining the $\partial_{z}$ component of $B$ shows that $u_{2}=u_{1}+(2 n+1) 2 K, n \in \mathbb{Z}$. Since $r^{2}$ is linearly related to $\kappa^{2}$ as before, by

$$
r^{2}=\left(\operatorname{dn}^{2} u-\frac{\sigma^{2}}{4 \mu^{2}}\right) /\left(4 a^{2} \mu^{2}\right)
$$

$r$ is the same at each end. Substituting (8) into $B\left(u_{2}\right)=-B\left(u_{1}\right)$ shows that either the first two components of $B\left(u_{1}\right)$ are zero (so that we can assume $u_{1}=0$ and $\sigma=1 / p)$, or $\theta\left(u_{2}\right)=\theta\left(u_{1}\right)-2 m \pi, m \in \mathbb{Z}$. Then the endpoints are vertically above each other, and the first two components of $B$ must again be zero at the endpoints.

In general, Type 2 curves are determined by integrating

$$
\begin{aligned}
& \frac{d z}{d s}=\frac{p^{-2}-\sigma^{2}-2 \mathrm{sn}^{2} u}{4 \mu}, \\
& \frac{d \theta}{d u}=L-\frac{N}{1-M \operatorname{sn}^{2} u},
\end{aligned}
$$

where the constants $L, M$, and $N$ are given by $L=\sigma p\left(p^{-2}-\sigma^{2}-2\right) /(8 \mu), M=$ $16 \mu^{2} /\left(p^{-2}+\sigma^{2}\right)^{2}$, and $N=2 \mu p \sigma /\left(p^{-2}+\sigma^{2}\right)+L$. When $\sigma=1 / p$, these equations simplify to

$$
\frac{d z}{d s}=-p \operatorname{sn}^{2} u, \quad \frac{d \theta}{d u}=L=-p / 2 .
$$

The changes of these variables over a cycle in $u$ of length $2 K$ are given by

$$
\Delta z=2(E-K) /(p \tau), \quad \Delta \theta=-K p
$$

( $E=E(p)$ being the complete elliptic integral of the second kind). Assuming $\tau>0$, both of these are negative, since $E<K$.

Now suppose the curve passes through $2 n+1$ of these cycles between its endpoints. Then $(2 n+1) K p=2 m \pi$, and the vertical distance between the endpoints is given by

$$
(2 n+1) \Delta z=4 \pi m \frac{K-E}{K p^{2} \tau} .
$$

It is easy to check that $(K-E) /\left(K p^{2}\right)$ is a monotone increasing function of $p$, with lower bound $1 / 2$ (see 4], 112.02). Hence, the vertical distance between the endpoints must be at least $2 m \pi / \tau$. So, every nontrivial Type 2 curve satisfying these boundary conditions must have distance between the endpoints at least $2 \pi / \tau$. Hence, if the distance is prescribed to be less than $2 \pi / \tau$, no smooth embedded minimal curve of constant torsion $\tau$ satisfies the boundary conditions.

We should also note that, even when the distance is above the critical value, only countably many values for $p$ satisfy the rationality condition $K p / \pi=2 m /(2 n+1)$, determining, via (11), only countably many possible distances. 


\section{REFERENCES}

1. R. Bishop, There is more than one way to frame a curve, Am. Math. Monthly 82 (1975), 246-251. MR 51:6604

2. K. Brakke, The Surface Evolver, Experiment. Math. 1 (1992), \#2, 141-165. MR 93k:53006

3. R. Bryant, P. Griffiths, Reduction for constrained variational problems and $\int k^{2} / 2 d s, A m e r$. J. Math. 108 (1986) 525-570. MR 88a:58044

4. P. Byrd, M. Friedman, Handbook of Elliptic Integrals for Engineers and Physicists, Springer, 1953. MR 15:702a

5. A. Calini, T. Ivey, Bäcklund transformations and knots of constant torsion, to appear in $J$. Knot Theory and its Ramifications 7 (1998), 719-746.

6. C. Carathéodory, Untersuchungen über das Denaunaysche Problem der Variationsrechnung, Abh. Math. Sem. Hamburg 8 (1930), 32-55.

7. W.L. Chow, Über Systeme von linearen partiellen Differentialgleichungen erster Ordnung, Math. Annalen 117 (1939), 98-105. MR 1:313d

8. G. Darboux, Leçons sur la Théorie Générale des Surfaces, Gauthier-Villars, 1917.

9. P.A. Griffiths, Exterior Differential Systems and the Calculus of Variations, Birkhäuser, 1983. MR 84h:58007

10. L. Hsu, Calculus of Variations via the Griffiths formalism, J. Diff. Geom. 36 (1992), 551-589. MR 94a:58003

11. J. Langer, D. Singer, Lagrangian Aspects of the Kirchhoff Elastic Rod, SIAM Reviews 38 (1996), 605-618. MR 97h:73050

12. L.S. Pontrjagin, V.G. Boltyanskii, R.V.Gamkrelidze, E.F. Mischenko, The mathematical theory of optimal processes, Wiley-Interscience, 1962. MR 29:3316b

13. R. Strichartz, Sub-Riemannian Geometry, J. Diff. Geom. 24 (1983), 221-263. MR 88b:53055

Department of Mathematical Sciences, Ball State University, Muncie, Indiana 47306

E-mail address: tivey@math.bsu.edu 